\title{
In-utero treatment of fetal asphyxia without C-section by the solution of placental fibrin deposit with heparin
}

\author{
Kazuo Maeda ${ }^{1 *}$ and Masaji Utsu \\ ${ }^{1}$ Obstetrics \& Gynecology, Honorary Professor, Tottori university Medical School, Yonago, Japan \\ ${ }^{2}$ Department of Obstetrics and Gynecology, Seirei-Mikatahara Hospital, Hamamatsu, Japan
}

\begin{abstract}
The gray level histogram width (GLHW) is an ultrasound tissue characterization, simply obtained from the histogram commonly prepared in the ultrasound B-mode devices. The GLHW has analysed various tissue problrms in Obstetrics and Gynecology. The GLHW diagnosed placental intervillous space fibrin deposit, which caused fetal growth restriction (FGR) and fetal asphyxia due to intervillous blood flow reduction, where the fibrin deposit was soluted by maternal heparin therapy achieving normal neonate without damage. Fetal asphyxa will be treated in the uterus, maybe without maternal C-sction in NRFS.
\end{abstract}

Key words: Feal asphyxia, Fibrin deposit, Placenta, FGR, GLHW, Heparin therapy, No NRFS, Normal baby

\section{Introduction}

Fetal asphyxia has been usually treated by early delivery with cesarean section (C-section). The author thought to treat fetal asphyxia keeping the fetus in the uterus without C- section. Placental insufficiency was discussed but its developing mechanism was rarely known, where decreased oxygen acquiring function of placental villi was the problem, but only villi dysplasia was found in preeclampsia by scanning electron microscopy [1]. Thus, the author studied oxygen transfer function of chorionic villi in fetal growth restriction (FGR). The fibrin deposit in intervillous space was reported to cause FGR and hypoxia, where its developing mechanism was studied with gray level histogram (GLHW) ultrasonic tissue characterization [2].

\section{Methods}

\section{Solution of fibrin deposit in intervillous space of placenta}

The fetal growth restriction (FGR) was diagnosed by reduced fetal weight in the $2^{\text {nd }}$ trimester of pregnancy, and the fibrin deposit in the placenta was diagnosed by the FGR and increased placental GLHW value calculated by the echogenicity histogram width divided by full 64 bits length of the histogram displayed in common ultrasound B-mode devices (Figure 1), which was standardized using RMI412 ultrasound phantom and various ultrasound B-mode imaging devices. Automated GLHW value was obtained by "\%W" index in the histogram of Aloka B-mode imaging device [2].

The GLHW value was stable if device gain changed, while it increased if the image contrast was high, therefore, the contrast of B-mode device was set the lowest in GLHW determination.

GLHW value was high also in malignancy, fibroid, fetal periventricular echo density, meconium stained amniotic fluid, while it was low in immature fetal lung [3].

As cardiolipine antigen was positive, an FGR patient who showed high placental GLHW was diagnosed as placental intervillous space fibrin deposit (Figure 2) and treated by the drip-infusion of $5000 \mathrm{U}$ heparin-Na every day from 17 to 31 gestational week.

\section{Results}

The technique would prevent fetal hypoxia, because GLHW value became lower, estimated fetal weight increased to normal, no asphyxic FHR change appeared, and normal neonate was achieved after the heparin treatment of the fibrin deposit FGR case (Figure 3), while the fetus of no heparin treatment was died in previous pregnancy of the same mother [2].

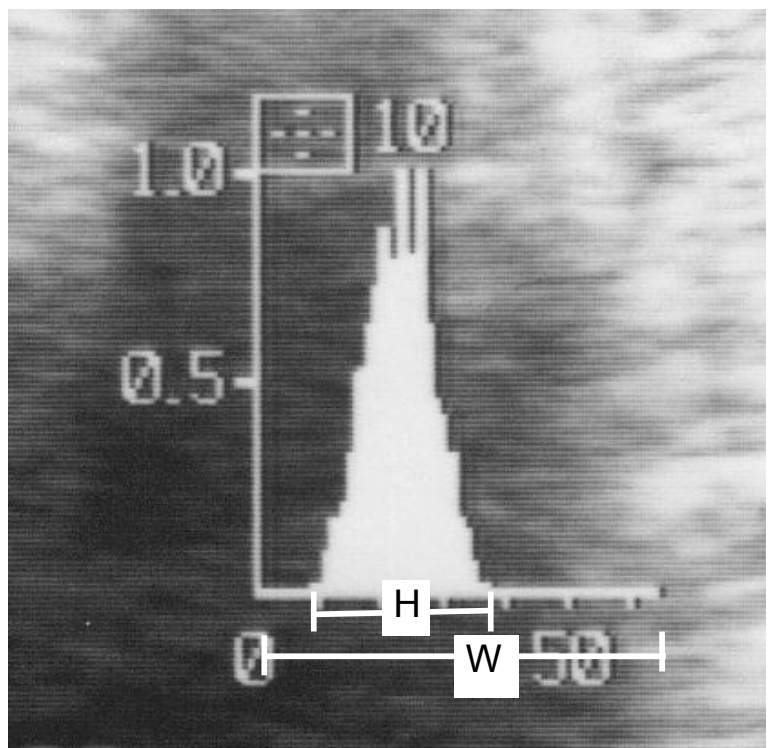

Figure 1. Measurement of GLHW on the echogenicity histogramof common ultrasound B-mode imaging device, where GLHW=H divided by W x 100 (\%).

Correspondence to: Kazuo Maeda, Obstetrics \& Gynecology, Honorary Professor, Tottori university Medical School, Yonago, Japan; Email: j2p4e4@ bma.biglobe.ne.jp

Received: April 28, 2017; Accepted: May 19, 2017; Published: May 22, 2017 


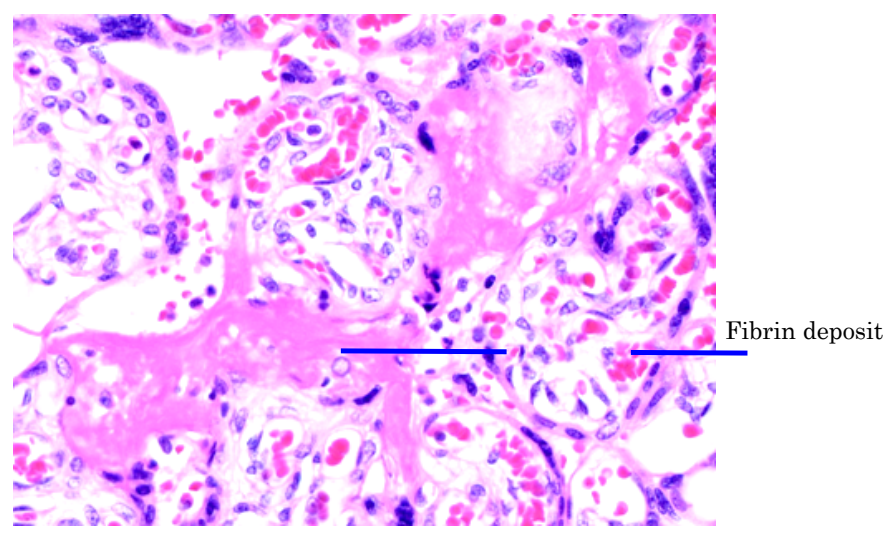

Figure 2. Microscopic view of a placental intervillous fibrin deposit. HE stain, 200x. Coutesy Utsu, M.

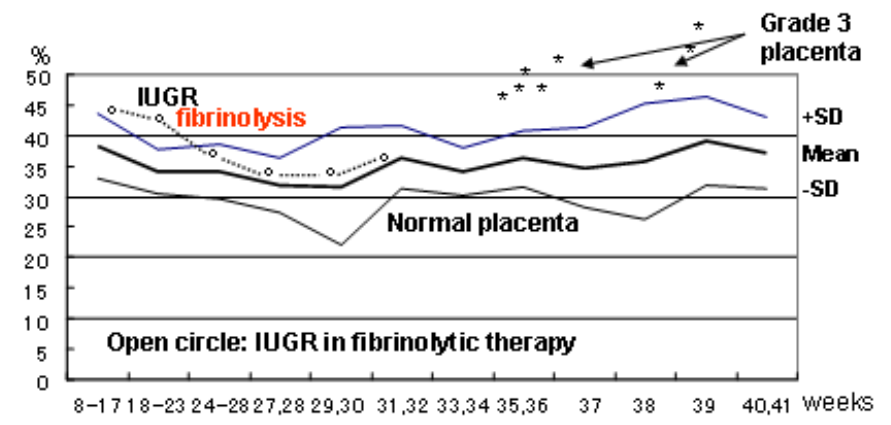

Figure 3. The GLHW value of IUGR (FGR) fetus was clearly lowered to normal level after the heparin treatment.

\section{Discussion}

Therefore, it was estimated that placental villi transfer of nutritive material was reduced by fibrin deposited FGR, while the transfer of smaller oxygen molecule was restricted in later developing hypoxia, which caused fetal demise in previous gestation of the same mother, who received no heparin treatment [2], where Utsu intended the solution of the fibrin deposit with heparin.

Initial NRFS was the loss of FHR acceleration in FGR preserving FHR variability, then some weeks later, heavy FHR changes appeared including severe late deceleration (LD). bradycardia and the loss of variability in untreated FGR. Although all cases of heavy asphyxia received C-section, neonatal depression was severe and some neonates died [5].

Therefore, initial loss of acceleration preserving variability was mild fetal hypoxia, and the loss of variability was severe fetal hypoxia damaging fetal brain [6]. The neurological sequel will be cerebral palsy. As heparin administration soluted fibrin deposit, placental blood flow was improved decreasing FGR and increasing fetal oxygenation by the heparin therapy, preventing Initial and advanced hypoxia, and normal neonate was achieved in the FGR case [2].

The theory was confirmed in another work on asphyxia in FGR, where 20 FGR cases of normally positive FHR acceleration and the other 20 FGR cases of no acceleration were compared. Positive acceleration cases maintained normal brain response to fetal movements, and no fetal brain damage was found. Another 20 FGR cases who lost FHR acceleration against grouped fetal motions (fetal movement burst). As FHR acceleration and variability were fetal brain responses to grouped and minor fetal movements, the loss of acceleration indicated initial mild hypoxia, and the loss of variability was advanced severe hypoxia, loosing whole fetal brain functions, simulating such heavy fetal brain damage as anencephaly [7].

Therefore, intervillous space fibrin deposit must be solved by heparin or thrombus lysing medicament, because the cause of FGR and hypoxia is fibrin deposit.

The fetus will be cured from the hypoxic damage by heparin in cases of FGR and high GLHW value, where abnormal pulsed Doppler flow of uterine artery may be associated. Heparin treatment was successful to prevent fetal death, which was occured in previous pregnancy of no heparin treatment in the same woman [3].

Fetal outcome would be assessed by the $\mathrm{A} / \mathrm{B}$ ratio value of actocardiogram (ACG), namely, outcome will be favorable if A/B ratio was larger than 1 in actocardiogram, and it was ominous if the $A / B$ ratio was less than $1[8]$.

Improving results of heparin therapy was shown in FGR case, in the reduction of GLHW value, increase of fetal weight, no hypoxic sign of FHR and fetal/neonatal deterioration. Those are the reason of authors recommendation to treat the fibrin deposit with heparin to treat the fetal distress or NRFS in-utero reducing C-section.

Heparin is not contraindicated if there is no hemorrhagic disease. Since possible effect of heparin is to solve fibrin to soluble fibrinogen, which is natural content of the serum, heparin will be safe to the mother and fetus, in non-hemorrhagic case.

\section{Conclusion}

A novel technique to treat in-utero fetal asphxia preventing fetal brain damage and fetal neonatal death would be maternal heparin therapy, which was proposed in the FGR associating high placental GLHW tissue characterization, where C-section will not be indicated but the birth would be vaginal delivery. Its effect will be confirmed before birth by increasing fetal weight, decreasing GLHW value and normal FHR records in the fetal monitoring.

\section{References}

1. Tsubaki O (1979) Scanning electron microscopic studies on the placental villi in toxemia of pregnancy. Acta Obstetrica Gynecol Jpn 31: 537

2. Maeda K, Utsu M, Kihaile PE (1998) Quantification of sonographic echogenicity with grey leel histogram width. A clinical tissue characterization. Ultrasound Med Biol 24: 225-234. [Crossref]

3. Maeda K (2005) Ultrasound tissue characterization with the gray level histogram width of the B-mode. Ultrasound Rev Obstet Gynecol 5: 92-95.

4. Serizawa M, Maeda K (2010) Non-invasive fetal lung maturity prediction based on ultrasonic gray level histogram width. Ultrasound Med Biol 36: 1998-2003.[Crossref]

5. Teshima N (1993) Non-reactive pattern diagnosed by ultrasonic Doppler fetal actocardiogram and outcome of the fetuses with non-reactive pattern. Acta Obstet Gynecol Jpn 45: 423-430. [Crossref]

6. Maeda K (2016) Fetal heart rate changes are the reaction of fetal brain to fetal movements in actocrdiogram: The loss of FHR variability is fetal brain damage. $J$ Preg Child Health.

7. Maeda K (2014) Modalities of fetal evaluation to detect fetal compromise prio to the development of significant neurological damage. J Obstet Gynaecol Res 40: 20892094. [Crossref]

8. Maeda K, Iwabe T, Yoshida S, Ito T, Minagawa Y et al. (2009) Detailed multigrade evaluation of fetal disorders with the quantified actocardiogram. J Perinat Med 37: 392-396. [Crossref]

Copyright: (C2017 Maeda K. This is an open-access article distributed under the terms of the Creative Commons Attribution License, which permits unrestricted use, distribution, and reproduction in any medium, provided the original author and source are credited. 